\title{
THE EQUIVALENCE OF TWO DEFINITIONS OF CAPACITY ${ }^{1}$
}

\author{
DAVID R. ADAMS AND JOHN C. POLKING
}

ABSTRACT. It is shown that two definitions for an $L_{p}$ capacity $(1<p<\infty)$ on subsets of Euclidean $\boldsymbol{R}^{n}$ are equivalent in the sense that as set functions their ratio is bounded above and below by positive finite constants. The classical notions of capacity correspond to the case $p=2$.

1. Let $L_{\alpha, p}=g_{\alpha}\left(L_{p}\right), 1<p<\infty, \alpha>0$, where $g_{\alpha}$ is the $L_{1}^{+}$function on $\boldsymbol{R}^{n}$ which is the Fourier transform of $(2 \pi)^{-n / 2}\left(1+|\xi|^{2}\right)^{-\alpha / 2}, \xi \in \boldsymbol{R}^{n} . L_{p}=$ $L_{p}\left(R^{n}\right)$ are the usual Lebesgue spaces.

DEFINITION 1. $B_{\alpha, p}(A)=\inf \|f\|_{p}^{p}$, where the infimum is over all $f \in L_{p}^{+}$ for which $g_{\alpha} * f(x) \geqq 1$ on $A, A \subset \boldsymbol{R}^{n}$.

DEFINITION 2. $C_{\alpha, p}(K)=\inf \|\varphi\|_{\alpha, p}^{p}$, where the infimum is over all $\varphi \in C_{0}^{\infty}\left(\boldsymbol{R}^{n}\right)$ for which $\varphi(x) \equiv 1$ in a neighborhood of $K, K$ compact set in $\boldsymbol{R}^{n}$.

Here $\|\cdot\|_{\alpha, p}$ denotes the usual norm in $L_{\alpha, p}$ and $\|\cdot\|_{0, p}=\|\cdot\|_{p}$. The purpose of this paper is to show

THEOREM A. For all compact sets $K \subset \boldsymbol{R}^{n}$,

$$
B_{\alpha, p}(K) \sim C_{\alpha, p}(K) .
$$

Here $\sim$ means that the ratio is bounded above and below by positive finite constants independent of the set $K$.

REMARK. The set function $C_{\alpha, p}$ is extended to the class of all subsets by $C_{\alpha, p}(A)=\sup C_{\alpha, p}(K)$, where the supremum is over all compact sets $K$ contained in $A$. When this is done, the equivalence of the theorem extends to all capacitable sets and in particular to all analytic sets. For details see [13].

As an example of the utility of these capacities, we state the following removable singularity theorem. Let $P(x, D)$ be a partial differential operator of order $m$ defined in an open set $\Omega \subset \boldsymbol{R}^{n}$.

Received by the editors July 29, 1971 and, in revised form, June 23, 1972.

AMS (MOS) subject classifications (1970). Primary 31C15, 26A33.

Key words and phrases. Capacity, Bessel potentials of $L_{p}$ functions, fractional differentiation operators, functions that operate.

${ }^{1}$ Research partially supported by National Science Foundation grant GP-23400.

c) American Mathematical Society 1973 
THEOREM B. Let $1<p<\infty$ and $1 / p+1 / q=1$. Let $A \subset \Omega$ be relatively closed.

(a) If $B_{m, q}(A)=0$, then each $f$ which is locally in $L_{p}(\Omega)$ and satisfies $P(x, D) f=0$ in $\Omega-A$, also satisfies $P(x, D) f=0$ in $\Omega$.

(b) Suppose $P(x, D)$ is elliptic and has a fundamental solution in $\Omega$. If each $f$, locally in $L_{p}(\Omega)$, which satisfies $P(x, D) f=0$ in $\Omega-A$ also satisfies $P(x, D) f=0$ in $\Omega$, then $B_{m, q}(A)=0$.

Theorem B is an easy corollary of Theorem A and the work of Littman [8], [9]. (See also [6].) As far as the authors know, Theorem B has not appeared explicitly in the literature though it seems to be widely known. For the case where $P(x, D)$ is a power of the Laplacian operator, Theorem $B$ is implicit in the work of Maz'ja and Havin [10]. Their removable singularity results are stated in terms of Hausdorff measure instead of capacity. These results and the $L^{p}$ removable singularity results in [5] follow directly from Theorem B and the relationship between $B_{\alpha, p}$ and Hausdorff measure (see [10], [13], or [16]).

The capacities defined above have been the subject of much investigation of late. $B_{\alpha, p}$ and closely related capacities have been studied by Fuglede [3], [4], Meyers [13], Adams and Meyers [1], and Rešetnjak [16], Maz'ja and Havin [10]. $C_{\alpha, p}$ has been studied by Littman [8], Maz'ja [12], and Harvey and Polking [6]. Maz'ja [11] has announced Theorem A in the case of integral $\alpha$.

To prove Theorem $\mathrm{A}$, we first notice that $B_{\alpha, p}(K) \leqq C_{\alpha, p}(K)$. This fact is elementary and only uses the fact that the kernels $g_{\alpha}$ are nonnegative. If $\varphi \in C_{0}^{\infty}\left(\boldsymbol{R}^{n}\right)$ and $\varphi(x)=1$ in a neighborhood of $K$, then $\varphi=g_{x} * \psi$ for some $\psi \in L_{p}$. Let $\psi_{+}(x)=\max (0, \psi(x))$. Then $\psi_{+} \in L_{p}^{+},\left\|\psi_{+}\right\|_{p} \leqq\|\psi\|_{p}=\|\varphi\|_{\alpha, p}$, and $g_{\alpha} * \psi_{+}(x) \geqq g_{\alpha} * \psi(x)=1$ for all $x \in K$.

To derive the reverse inequality, we use a method due to Littman [8]. The key fact that makes the method work is the boundedness principle for capacity extremals. The form that we will use is the following: Let $G$ be an open set in $\boldsymbol{R}^{n}$. Then there is a function $f \in L_{p}^{+}$such that: (a) $g_{\alpha} * f(x) \geqq 1$ on $G$, (b) $g_{\alpha} * f(x) \leqq Q<\infty$ for all $x \in \boldsymbol{R}^{n}$, where $Q$ is a constant depending only on $n$ and $p$, and (c) $\|f\|_{p}^{p}=B_{x, p}(G)$. (This result was obtained independently by Adams and Meyers [1] and Maz'ja and Havin [10].) So, for $K$ compact we choose such a $G \supset K$. Furthermore, we can find $G_{1}$ open: $K \subset G_{1} \subset G$ and a $C_{0}^{\infty}$ regularization $\varphi$ of $g_{\alpha} * f(f$ chosen as above for $G$ ) such that: $\left(\mathrm{a}^{\prime}\right) \varphi(x) \geqq 1$ on $G_{1},\left(\mathrm{~b}^{\prime}\right) 0 \leqq \varphi(x) \leqq Q$, for all $x$ (same $Q$ ), and (c') $\|\varphi\|_{\alpha, p} \leqq$ const $\|f\|_{p}$. Now let $H(t), t \geqq 0$, be a $C^{\infty}\left(\boldsymbol{R}_{+}^{1}\right)$ function such that $H(t)=t, 0 \leqq t \leqq \frac{1}{2}$, and $H(t)=1, t \geqq 1$. Clearly, $\|H(\varphi)\|_{p} \leqq$ const $\|\varphi\|_{p}$. The proof of the theorem will now be concluded by showing: for any $\alpha>0$,

$$
\|H(\varphi)\|_{\alpha, p} \leqq Q_{1}\|\varphi\|_{\alpha, p}\left(\sum_{j<\alpha}\|\varphi\|_{\infty}^{j}\right)
$$


for some constant $Q_{1}$ independent of $\varphi$. To see that (1) completes the proof, we need only observe that $H(\varphi) \equiv 1$ on $G_{1}, H(\varphi) \in C_{0}^{\infty}\left(\boldsymbol{R}^{n}\right)$ and

$$
C_{\alpha, p}(K) \leqq\|H(\varphi)\|_{\alpha, p}^{p} \leqq \text { const }\|f\|_{p}^{p}=\text { const } B_{\alpha, p}(G)
$$

by the known properties of $\varphi$. The desired inequality then follows by the capacitability results of [13]. Note the above const is independent of the sets $K$ and $G$.

Proof of (1). We consider two cases.

Case 1. $\alpha=$ integer. We need some preliminary theorems.

TheOREM 1 ([2]). For $1<p<\infty$ and $\alpha$ integral,

$$
\|\varphi\|_{\alpha, p} \sim \sum_{0 \leqq|s| \leqq \alpha}\left\|D^{s} \varphi\right\|_{p} .
$$

$D^{s}$ denotes derivatives in the coordinate directions $s=\left(s_{1}, \cdots, s_{n}\right),|s|=$ $s_{1}+\cdots+s_{n}$.

THEOREM 2. For $0<\lambda<1,0<\alpha<\infty$ ( $\alpha$ not necessarily integral),

$$
\|\varphi\|_{\alpha(1-\lambda), p /(1-\lambda)} \leqq c\|\varphi\|_{\alpha, p}^{1-\lambda}\|\varphi\|_{\alpha}^{\lambda}
$$

$c$ independent of $\varphi$.

For periodic functions on [0,2 $\pi$, this theorem is due to Hirschman [7]. For integral $\alpha$ and $\alpha(1-\lambda)$, it is the well-known inequality of Nirenberg and Gagliardo (see [14]). A proof of Theorem 2 may be found in [1].

Since for real $\beta, \gamma, 0 \leqq \beta \leqq \gamma,\|u\|_{\beta, p} \leqq\|u\|_{\gamma, p}$, we need only concentrate on estimating $D^{s} H(\varphi)$ in $L_{p},|s|=\alpha$. There are positive constants $C_{r^{1}, \ldots, r^{\prime}}$ depending only on the multi-index subscripts such that

$$
D^{s} H(\varphi)=\sum_{j=1}^{\alpha} H^{(j)}(\varphi) \sum C_{r^{1}, \cdots, r^{j}} D^{r^{1}} \varphi \cdots D^{r^{j}} \varphi,
$$

with the last sum over all sets of multi-indices $\left(r^{1}, \cdots, r^{j}\right)$, such that $\left|r^{i}\right| \geqq 1$ and $r^{1}+\cdots+r^{j}=s$. Hölder's inequality gives

$$
\left\|\prod_{l=1}^{j} D^{r^{l}} \varphi\right\|_{p} \leqq \prod_{l=1}^{j}\left\|D^{r^{l}} \varphi\right\|_{p_{p_{l}}},
$$

$\sum_{l}\left(1 / p_{l}\right)=1,1<p_{l}<\infty$. Choose $p_{l}=\alpha /\left|r^{l}\right|$. Then

$$
\left\|D^{r^{l}} \varphi\right\|_{p n_{l}} \leqq c\|\varphi\|_{\alpha / p_{l}, p p_{l}} \leqq c\|\varphi\|_{\alpha, p}^{1 / p_{l}}\|\varphi\|_{\infty}^{1-1 / \nu_{l}}
$$


Case 2. $\alpha \neq$ integer. The preliminary results needed here are

THEOREM 3. $\varphi \in L_{\alpha, p}, \alpha>1$, if and only if $D^{s} \varphi \in L_{\alpha-|s| . p}, 0 \leqq|s| \leqq[\alpha]$, and

$$
\|\varphi\|_{\alpha, p} \sim \sum_{0 \leqq|s| \leqq[\alpha]}\left\|D^{s} \varphi\right\|_{\alpha-[\alpha] . p}
$$

Here $[\alpha]=$ greatest integer in $\alpha$.

Theorem 3 is an easy corollary of Theorem 1 and the definition of the space $L_{\alpha, p}$.

Let

Then

$$
\mathscr{D}_{p}^{\alpha}(u)(x)=\left(\int_{0}^{\infty}\left(\int_{|y| \leqq 1}|u(x+\rho y)-u(x)|^{D} d y\right)^{2} \frac{d \rho}{\rho^{1+2 x p}}\right)^{1 / 2 D} .
$$

THEOREM 4. (a) ([15]). If $1 \leqq p<\infty, 0<\alpha<1$, and $\max (1, n p /(n+\alpha p))<$ $r<\infty$, then $\left\|\mathscr{D}_{p}^{\alpha}(u)\right\|_{r} \leqq c\|u\|_{\alpha . r}$, all $u \in L_{\alpha, r}$.

(b) ([17]) $\left\|\mathscr{D}_{1}^{\alpha}(u)\right\|_{r}+\|u\|_{r} \sim\|u\|_{\alpha, r}, 1<r<\infty, 0<\alpha<1$.

We begin with $0<\alpha<1$.

$$
\begin{aligned}
\|H(\varphi)\|_{\alpha, p} & \leqq c\left\|\mathscr{D}_{1}^{\alpha} H(\varphi)\right\|_{p}+c\|H(\varphi)\|_{p} \\
& \leqq c\left\|\mathscr{D}_{1}^{\alpha}(\varphi)\right\|_{p}+c\|\varphi\|_{p} \leqq c\|\varphi\|_{\alpha, p} .
\end{aligned}
$$

Note $\mathscr{D}_{1}^{\alpha} H(\varphi) \leqq c \mathscr{D}_{1}^{\alpha}(\varphi)$ since $H$ is Lipschitz continuous.

Now assume $\alpha=k+\sigma, k$ a positive integer and $0<\sigma<1$.

$$
\|H(\varphi)\|_{\alpha, p} \leqq c \sum_{0 \leqq|s| \leqq k}\left\|D^{s} H(\varphi)\right\|_{\sigma, p}
$$

Again we need only consider the case $|s|=k$.

$$
\left\|D^{s} H(\varphi)\right\|_{\sigma, p} \leqq c\left\|\mathscr{D}_{1}^{\sigma}\left(D^{s} H(\varphi)\right)\right\|_{p}+c\left\|D^{s} H(\varphi)\right\|_{p} .
$$

The last term has already been treated. Now using (2) with $\alpha$ replaced by $k$, it easily follows that

$$
\mathscr{D}_{1}^{\sigma}\left(D^{s} H(\varphi)\right) \leqq c \mathscr{D}_{1}^{\sigma}\left(D^{r^{1}} \varphi \cdots D^{r^{j}} \varphi\right)+c\left|D^{r^{1}} \varphi \cdots D^{r^{j}} \varphi\right| \mathscr{D}_{1}^{\sigma}(\varphi),
$$

again noting $\mathscr{D}_{1}^{\sigma}\left(H^{(j)}(\varphi)\right) \leqq c \mathscr{D}_{1}^{\sigma}(\varphi)$. The $L_{p}$ norm of the last term in the above sum does not exceed $c\left\|D^{r^{1}} \varphi\right\|_{\alpha p /\left|r^{1}\right|} \cdots\left\|D^{r^{j}} \varphi\right\|_{\alpha p /\left|r^{j}\right|}\left\|\mathscr{D}_{1}^{\sigma}(\varphi)\right\|_{\alpha p / \sigma} \leqq$ $c\|\varphi\|_{\alpha, p}\|\varphi\|_{\infty}^{j}$, upon applying Hölder's inequality and Theorems 4,1 and 2 .

To treat the remaining terms, we use

TheOREM 5 ([15]). If $0<\lambda, \mu<1$, then

$$
\mathscr{D}^{\sigma}(f g) \leqq|f| \mathscr{D}_{p}^{\sigma}(g)+|g| \mathscr{D}_{p}^{\sigma}(f)+\mathscr{D}_{p / \mu}^{\lambda \sigma}(f) \mathscr{D}_{p /(1-, .)}^{(1-\lambda) \sigma}(g) .
$$


Using the convention $\mathscr{D}_{\infty}^{0}(f)=|f|$, repeated applications of Theorem 5 to $\mathscr{D}_{1}^{\sigma}\left(D^{r^{1}} \varphi \cdots D^{r^{j}} \varphi\right)$ show that this expression is bounded by a finite sum of terms of the form

$$
\mathscr{D}_{q_{1}}^{\sigma_{j}}\left(D^{r^{1}} \varphi\right) \cdots \mathscr{D}_{q_{j}}^{\sigma_{j}}\left(D^{r^{j}} \varphi\right) .
$$

The number of terms depends only on $j$. Here $\sigma_{i} \geqq 0$ and $q_{i} \geqq 1\left(q_{k}=\infty\right.$ when and only when $\left.\sigma_{i}=0\right)$, and $\sum \sigma_{i}=\sigma, \sum\left(1 / q_{i}\right)=1$. In addition, Theorem 5 allows us considerable freedom in choosing the nonzero $\sigma_{i}$ 's and finite $q_{i}$ 's; this permits us to make a particular choice so that Theorem 4(a) applies below. Namely, we choose $q_{i} \leqq \alpha /\left(\sigma_{i}+\left|r^{i}\right|\right)$ when $q_{i}<\infty$. Now setting $p_{i}=\alpha p /\left(\sigma_{i}+\left|r^{i}\right|\right)$, we have, applying Hölder's inequality together with Theorems 4,3 and 2, that the $L_{p}$ norm of (3) does not exceed

$$
\begin{aligned}
\prod_{i=1}^{j}\left\|\dot{\mathscr{X}}_{q i}^{\sigma_{i}}\left(D^{r^{i}} \varphi\right)\right\|_{p_{i}} & \leqq c \prod\left\|D^{r^{i}} \varphi\right\|_{\sigma_{i}, p_{i}} \leqq c \prod\|\varphi\|_{\sigma_{i}+\left|r^{i}\right|, p_{i}} \\
& \leqq c\|\varphi\|_{\alpha, p}\|\varphi\|_{\infty}^{j-1} .
\end{aligned}
$$

\section{REFERENCES}

1. D. R. Adams and N. G. Meyers, Bessel potentials. Inclusion relations among classes of exceptional sets, Indiana Univ. Math. J. (to appear). (An announcement of these results appears in Bull. Amer. Math. Soc. 77 (1971), 968-970.)

2. A. P. Calderón, Lebesgue spaces of differentiable functions and distributions, Proc. Sympos. Pure Math., vol. 4, Amer. Math. Soc., Providence, R.I., 1961, pp. 33-49. MR $26 \# 603$.

3. B. Fuglede, Extremal length and functional completion, Acta Math. 98 (1957), 171-219. MR 20 \#4187.

4. - Application du théorème minimax a l'étude diverse capacités, C. R. Acad. Sci. Paris 266 (1968), 921-923.

5. R. Harvey and J. C. Polking, Removable singularities of solutions of linear partial differential equations, Acta Math. 125 (1970), 39-56. MR 43 \#5183.

6. - A notion of capacity which characterizes removable singularities, Trans. Amer. Math. Soc. 169 (1972), 183-195.

7. I. I. Hirschman, $A$ convexity theorem for certain groups of transformations, J. Analyse Math. 2 (1952/53), 209-218. MR 15, $295 ; 1139$.

8. W. Littman, $A$ connection between $\alpha$-capacity and m-p polarity, Bull. Amer. Math. Soc. 73 (1967), 862-866. MR 36 \#2940.

9. - Polar sets and removable singularities of partial differential equations, Ark. Mat. 7 (1967), 1-9. MR 37 \#559.

10. V. G. Maz'ja and V. P. Havin, A nonlinear analogue of the Newtonian potential and metric properties of $(p, 1)$-capacity, Dokl. Akad. Nauk SSSR 194 (1970), 770 773 =Soviet Math. Dokl. 11 (1970), 1294-1298. MR 42 \#7926.

11. V. G. Maz'ja, Imbedding theorems and their applications, Baku Sympos. (1966), "Nauka", Moscow, 1970, pp. 142-159. (Russian).

12. - p-conductance and theorems of imbedding certain function spaces into the space $\mathfrak{C}$, Dokl. Akad. Nauk SSSR 140 (1961), 299-302=Soviet Math. Dokl. 2 (1961), 1200-1203. MR 28 \#460. 
13. N. G. Meyers, A theory of capacities for potentials of functions in Lebesgue classes, Math. Scand. 26 (1970), 255-292. MR 43 \#3474.

14. L. Nirenberg, On elliptic partial differential equations, Ann. Scuola Norm. Sup. Pisa (3) 13 (1959), 115-162. MR 22 \#823.

15. J. C. Polking, A Leibniz formula for some differentiation operators of fractional order, Indiana Univ. Math. J. 21 (1972), 1019-1029.

16. Ju. G. Rešetnjak, The concept of capacity in the theory of functions with generalized derivatives, Sibirsk. Mat. Ž. 10 (1969), 1109-1138=Siberian Math. J. 10 (1969), 818842. MR 43 \#2234.

17. R. S. Strichartz, Multipliers on fractional Sobolev spaces, J. Math. Mech. 16 (1967), 1031-1060. MR 35 \#5927.

Department of Mathematics, Rice University, Houston, Texas 77001 (Current address of John C. Polking)

Current address (David R. Adams): Department of Mathematics, University of California at San Diego, La Jolla, California 92037 